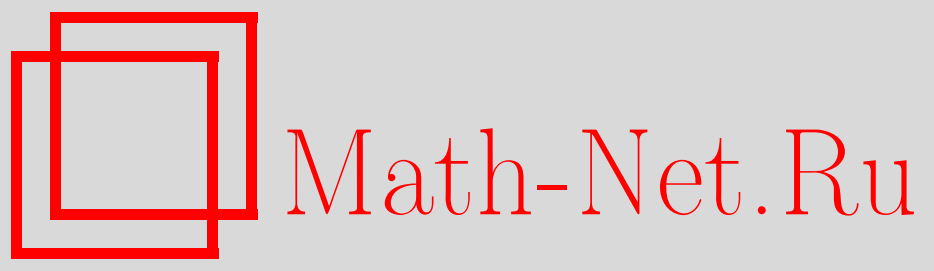

У. А. Розиков, М. М. Рахматуллаев, Описание слабо периодических мер Гиббса модели Изинга на дереве Кэли, TMФ, 2008, том 156, номер 2, 292-302

DOI: https://doi.org/10.4213/tmf6248

Использование Общероссийского математического портала Math-Net.Ru подразумевает, что вы прочитали и согласны с пользовательским соглашением http://www . mathnet.ru/rus/agreement

Параметры загрузки:

IP: 54.197 .130 .99

26 апреля 2023 г., 14:29:56

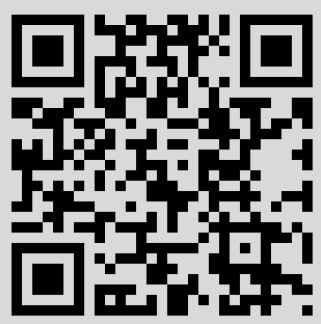




\title{
ОПИСАНИЕ СЛАБО ПЕРИОДИЧЕСКИХ МЕР ГИББСА МОДЕЛИ ИЗИНГА НА ДЕРЕВЕ КЭЛИ
}

\begin{abstract}
Введено понятие слабо периодической меры Гиббса. Для модели Изинга описано множество таких мер, соответствующих нормальным делителям индекса 2 и индекса 4 группового представления дерева Кэли. В частности, на дереве Кэли порядка 4 доказано, что существуют критические значения $T_{\mathrm{c}}<T_{\text {сr }}$ тем-

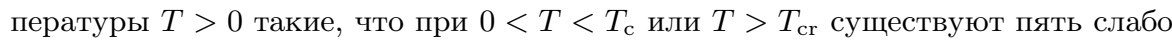
периодических мер Гиббса, при $T=T_{\text {c }}$ существуют три слабо периодических меры Гиббса, при $T_{\mathrm{c}}<T \leqslant T_{\text {cr }}$ существует одна слабо периодическая мера Гиббса.
\end{abstract}

Ключевые слова: дерево Кэли, мера Гиббса, модель Изинга, слабо периодические меры.

\section{1. ВВЕДЕНИЕ}

Одна из основных проблем для гамильтониана модели Изинга - это описание всех отвечающих ему предельных мер Гиббса. Известно, что для модели Изинга такие меры образуют непустое выпуклое компактное подмножество в множестве всех вероятностных мер. Задача полного описания элементов этого множества далека от своего завершения. Описаны трансляционно-инвариантные (см., например, [1]-[3]), периодические [4], [5] и континуальные множества непериодических [1], [6] мер Гиббса для модели Изинга на дереве Кэли.

Описанию периодических мер Гиббса для некоторых моделей с конечным радиусом взаимодействия посвящены работы [4], [5], [7]-[11], в которых в основном рассматриваются трансляционно-инвариантные или периодические с периодом два меры. В настоящей работе мы введем более общее понятие периодической меры Гиббса и проверим существование таких мер для модели Изинга.

Структура работы такова. В разделе 2 мы даем необходимые определения и постановку задачи. Раздел 3 посвящен описанию слабо периодических мер. В

${ }^{*}$ Институт математики и информационных технологий АН РУз, Ташкент, Узбекистан. E-mail: rozikovu@yandex.ru

${ }^{\dagger}$ Наманганский государственный университет, Наманган, Узбекистан. E-mail: mrahmatullaev@rambler.ru 
п. 3.1 изучаются слабо периодические меры, соответствующие нормальным делителям индекса 2, а в п. 3.2 рассматривается один нормальный делитель индекса 4, выбор которого обеспечивает минимум числа неизвестных в соответствующей системе уравнений. В разделе 4 мы обсуждаем полученные результаты и формулируем несколько проблем, оставшихся открытыми.

\section{2. ОПРЕДЕЛЕНИЯ И ПОСТАНОВКА ЗАДАЧИ}

Пусть $\tau^{k}=(V, L), k \geqslant 1$, есть дерево Кэли порядка $k$, т.е. бесконечное дерево, из каждой вершины которого выходит ровно $k+1$ ребер, $V$ - множество вершин, $L-$ множество ребер $\tau^{k}$. Известно, что $\tau^{k}$ можно представить как $G_{k}$ - свободное произведение $k+1$ циклических групп второго порядка с образующими $a_{1}, a_{2}, \ldots, a_{k+1}$.

Для произвольной точки $x^{0} \in V$ положим $W_{n}=\left\{x \in V \mid d\left(x^{0}, x\right)=n\right\}, V_{n}=$ $\bigcup_{m=0}^{n} W_{m}, L_{n}=\left\{\langle x, y\rangle \in L \mid x, y \in V_{n}\right\}$, где $d(x, y)$ - расстояние между $x$ и $y$ на дереве Кэли, т.е. число ребер пути, соединяющего $x$ и $y$ (здесь и далее $\langle x, y\rangle$ означает, что вершины $x$ и $y$ являются ближайшими соседями).

Пусть $\Phi=\{-1,1\}$ и $\sigma$ - конфигурация на $V$, т.е. $\sigma=\{\sigma(x) \in \Phi: x \in V\}, \Omega=\Phi^{V}$. Пусть $A \subset V$. Обозначим через $\Omega_{A}$ пространство конфигураций, определенных на множестве $A$ и принимающих значения из $\Phi$.

Рассмотрим гамильтониан модели Изинга

$$
H(\sigma)=-J \sum_{\langle x, y\rangle \in L} \sigma(x) \sigma(y), \quad J \in \mathbb{R} .
$$

Пусть $h_{x} \in \mathbb{R}, x \in V$. Для каждого $n$ определим меру $\mu_{n}$ на $\Omega_{V_{n}}$, полагая

$$
\mu_{n}\left(\sigma_{n}\right)=Z_{n}^{-1} \exp \left\{-\beta H\left(\sigma_{n}\right)+\sum_{x \in W_{n}} h_{x} \sigma(x)\right\},
$$

где $\beta=T^{-1}$ - обратная температура, $T>0, \sigma_{n}=\left\{\sigma(x), x \in V_{n}\right\} \in \Omega_{V_{n}}, Z_{n}^{-1}-$ нормировочный множитель и

$$
H\left(\sigma_{n}\right)=-J \sum_{\langle x, y\rangle \in L_{n}} \sigma(x) \sigma(y) .
$$

Условия согласованности для последовательности мер $\mu_{n}\left(\sigma_{n}\right), n \geqslant 1$, задаются равенством

$$
\sum_{\sigma^{(n)}} \mu_{n}\left(\sigma_{n-1}, \sigma^{(n)}\right)=\mu_{n-1}\left(\sigma_{n-1}\right), \quad \sigma^{(n)}=\left\{\sigma(x), x \in W_{n}\right\} .
$$

Пусть $\mu_{n}, n \geqslant 1,-$ последовательность мер на $\Omega_{V_{n}}$, обладающая свойством согласованности (3). Тогда в силу теоремы Колмогорова существует, и притом единственная, предельная мера $\mu$ на $\Omega_{V}=\Omega$ (которая называется предельной мерой Гиббса) такая, что $\mu\left(\sigma_{n}\right)=\mu_{n}\left(\sigma_{n}\right)$ для каждого $n=1,2, \ldots$ Известно, что меры (2) удовлетворяют (3) тогда и только тогда, когда совокупность величин $h=\left\{h_{x}, x \in G_{k}\right\}$ такова, что

$$
h_{x}=\sum_{y \in S(x)} f\left(h_{y}, \theta\right)
$$


где $S(x)$ - множество “прямых потомков" точки $x \in V$ и $f(x, \theta)=\operatorname{arcth}(\theta \operatorname{th} x)$, $\theta=\operatorname{th}(J \beta)($ cм. [1]-[3]).

ОПРЕДЕЛЕНИЕ 1. Совокупность величин $h=\left\{h_{x}, x \in G_{k}\right\}$ называется $\widehat{G}_{k}$-nериодической, если $h_{x y}=h_{x}$ для любых $x \in G_{k}, y \in \widehat{G}_{k}$ (здесь $\widehat{G}_{k}-$ нормальный делитель индекса $r \geqslant 1$ ).

В настоящей работе мы дадим более общее определение периодической меры Гиббса. Для $x \in G_{k}$ положим $x_{\downarrow}=\left\{y \in G_{k}:\langle x, y\rangle\right\} \backslash S(x)$. Пусть $G_{k} / \widehat{G}_{k}=$ $\left\{H_{1}, \ldots, H_{r}\right\}-$ фактор-группа.

ОПРЕДЕЛЕНИЕ 2. Совокупность величин $h=\left\{h_{x}, x \in G_{k}\right\}$ назовем $\widehat{G}_{k}$-слабо периодической, если $h_{x}=h_{i j}$ при $x \in H_{i}, x_{\downarrow} \in H_{j}$ для любого $x \in G_{k}$.

Заметим, что слабо периодическая совокупность $h$ совпадает с обычной периодической (см. определение 1$)$, если значение $h_{x}$ не зависит от $x_{\downarrow}$.

ОПРЕДЕЛЕНИЕ 3 . Меру $\mu$ назовем $\widehat{G}_{k^{-}}($слабо) периодической, если она соответствует $\widehat{G}_{k^{-}}$(слабо) периодической совокупности величин $h . G_{k}$-периодическая мера называется трансляиионно-инвариантной.

Цель нашей работы - описание множества слабо периодических мер Гиббса для модели Изинга.

\section{3. СЛАБО ПЕРИОДИЧЕСКИЕ МЕРЫ}

Степень трудности задачи описания слабо периодических мер Гиббса зависит от структуры и индекса нормального делителя, относительно которого налагается условие периодичности. В работе [12] доказано, что в группе $G_{k}$ не существует нормального делителя нечетного индекса, отличного от 1. Поэтому мы рассмотрим нормальные делители четного индекса. В настоящей работе мы ограничимся случаем индексов 2 и 4.

3.1. Случай индекса 2. Опишем $\bar{G}_{k}$-слабо периодические меры Гиббса для любого нормального делителя $\bar{G}_{k}$ индекса 2. Заметим, что любой нормальный делитель индекса 2 группы $G_{k}$ имеет вид $H_{A}=\left\{x \in G_{k}: \sum_{i \in A} w_{x}\left(a_{i}\right)\right.$ - четное число $\}$, где $\varnothing \neq A \subseteq N_{k}=\{1,2, \ldots, k+1\}$ и $w_{x}\left(a_{i}\right)$ - число букв $a_{i}$ в слове $x \in G_{k}$ [4].

Пусть $A \subset N_{k}$ и $H_{A}-$ соответствующий нормальный делитель индекса 2. Заметим, что в случае $|A|=k+1(|A|$ - число элементов множества $A)$, т.е. в случае $A=N_{k}$, слабая периодичность совпадает с обычной периодичностью. Поэтому рассмотрим $A \subset N_{k}$ такие, что $A \neq N_{k}$. Тогда в силу (4) $H_{A}$-слабо периодическая совокупность $h$ имеет вид

$$
h_{x}= \begin{cases}h_{1}, & x \in H_{A}, \quad x_{\downarrow} \in H_{A}, \\ h_{2}, & x \in H_{A}, \quad x_{\downarrow} \in G_{k} \backslash H_{A}, \\ h_{3}, & x \in G_{k} \backslash H_{A}, \quad x_{\downarrow} \in H_{A}, \\ h_{4}, & x \in G_{k} \backslash H_{A}, \quad x_{\downarrow} \in G_{k} \backslash H_{A},\end{cases}
$$


где $h_{i}, i=\overline{1,4}$, удовлетворяют системе уравнений

$$
\begin{aligned}
& h_{1}=|A| f\left(h_{3}, \theta\right)+(k-|A|) f\left(h_{1}, \theta\right), \\
& h_{2}=(|A|-1) f\left(h_{3}, \theta\right)+(k+1-|A|) f\left(h_{1}, \theta\right), \\
& h_{3}=(|A|-1) f\left(h_{2}, \theta\right)+(k+1-|A|) f\left(h_{4}, \theta\right), \\
& h_{4}=|A| f\left(h_{2}, \theta\right)+(k-|A|) f\left(h_{4}, \theta\right) .
\end{aligned}
$$

Рассмотрим отображение $W: \mathbb{R}^{4} \rightarrow \mathbb{R}^{4}$, определенное следующим образом: $W(h)=h^{\prime}$, если

$$
\begin{aligned}
h_{1}^{\prime} & =|A| f\left(h_{3}, \theta\right)+(k-|A|) f\left(h_{1}, \theta\right), \\
h_{2}^{\prime} & =(|A|-1) f\left(h_{3}, \theta\right)+(k+1-|A|) f\left(h_{1}, \theta\right), \\
h_{3}^{\prime} & =(|A|-1) f\left(h_{2}, \theta\right)+(k+1-|A|) f\left(h_{4}, \theta\right), \\
h_{4}^{\prime} & =|A| f\left(h_{2}, \theta\right)+(k-|A|) f\left(h_{4}, \theta\right) .
\end{aligned}
$$

Заметим, что система (6) эквивалентна уравнению $h=W(h)$. Отображение $W$ имеет инвариантные множества

$$
\begin{gathered}
I_{1}=\left\{h \in \mathbb{R}^{4}: h_{1}=h_{2}=h_{3}=h_{4}\right\}, \quad I_{2}=\left\{h \in \mathbb{R}^{4}: h_{1}=h_{4}, h_{2}=h_{3}\right\}, \\
I_{3}=\left\{h \in \mathbb{R}^{4}: h_{1}=-h_{4}, h_{2}=-h_{3}\right\} .
\end{gathered}
$$

Пусть $\alpha=(1-\theta) /(1+\theta)$.

Теорема 1. Справедливы следующие утверждения.

1. Для модели Изинга все $H_{A}$-слабо периодические меры Гиббса на $I_{1}, I_{2}$ являются трансляционно-инвариантными.

2. При $|A|=k$ и $\theta>0$ все $H_{A}$-слабо периодические мерь Гиббса являются трансляционно-инвариантными.

3. При $|A|=1, k=4$ существует критическое значение $\alpha_{\mathrm{cr}}(\approx 0.152)$ такое, что при $0<\alpha<\alpha_{\text {cr }}$ существуют пять $H_{A}$-слабо периодических мер Гиббса $\mu_{0}$, $\mu_{1}^{ \pm}, \mu_{2}^{ \pm} ;$при $\alpha=\alpha_{\text {сr }}$ существуют три $H_{A^{-}}$-слабо периодические меры Гиббса $\mu_{0}, \mu_{1}^{ \pm}$; при $\alpha>\alpha_{\mathrm{cr}}$ существует единственная $H_{A}$-слабо периодическая мера Гиббса $\mu_{0}$.

4. При $|A|=1, k>5$ и $\theta_{-}<\theta<\theta_{+}, \theta_{ \pm}=\left(k-1 \pm \sqrt{k^{2}-6 k+1}\right) / 2 k$, на I существуют три $H_{A}$-слабо периодические мерь Гиббса $\mu^{0}, \mu^{ \pm}$.

ДоказАтельство. 1. Достаточно показать, что система уравнений (6) имеет только корень вида $h_{1}=h_{2}=h_{3}=h_{4}$. Для инвариантного множества $I_{1}$ доказательство очевидно. Докажем это утверждение для инвариантного множества $I_{2}$.

Пользуясь тем, что

$$
f(h, \theta)=\operatorname{arcth}(\theta \operatorname{th} h)=\frac{1}{2} \ln \frac{(1+\theta) e^{2 h}+(1-\theta)}{(1-\theta) e^{2 h}+(1+\theta)},
$$


и вводя обозначения $z_{i}=e^{2 h_{i}}, i=\overline{1,4}$, вместо (6) получим следующую систему уравнений:

$$
\begin{aligned}
z_{1}-z_{2} & =A_{1}\left(z_{3}-z_{1}\right) \\
z_{1}-z_{3} & =A_{2}\left(z_{1}-z_{4}\right)+B_{2}\left(z_{3}-z_{4}\right)+C_{2}\left(z_{3}-z_{2}\right) \\
z_{1}-z_{4} & =A_{3}\left(z_{1}-z_{4}\right)+B_{3}\left(z_{3}-z_{2}\right) \\
z_{2}-z_{3} & =A_{4}\left(z_{3}-z_{2}\right)+B_{4}\left(z_{1}-z_{4}\right) \\
z_{2}-z_{4} & =A_{5}\left(z_{3}-z_{2}\right)+B_{5}\left(z_{1}-z_{2}\right)+C_{5}\left(z_{1}-z_{4}\right) \\
z_{3}-z_{4} & =A_{6}\left(z_{4}-z_{2}\right)
\end{aligned}
$$

где $A_{i}=\left(1-\alpha^{2}\right) \tilde{A}_{i}\left(z_{1}, z_{2}, z_{3}, z_{4}\right), B_{i}=\left(1-\alpha^{2}\right) \widetilde{B}_{i}\left(z_{1}, z_{2}, z_{3}, z_{4}\right), C_{i}=\left(1-\alpha^{2}\right) \times$ $\widetilde{C}_{i}\left(z_{1}, z_{2}, z_{3}, z_{4}\right)$ и $\tilde{A}_{i}, \widetilde{B}_{i}, \widetilde{C}_{i}$ положительны для всех $i=\overline{1,6}$.

На инварианте $I_{2}$ мы имеем $h_{2}=h_{3}$, откуда при $\alpha<1$ из $z_{1}-z_{2}=A_{1}\left(z_{3}-z_{1}\right)$ следует, что $z_{1}=z_{2}$.

В антиферромагнитном случае, т.е. при $\alpha>1$, получаем что $A_{i}, B_{i}, C_{i}<0$ для всех $i=\overline{1,6}$. На инварианте $I_{2}$ выполняется равенство $h_{2}=h_{3}$. Из (8) имеем $z_{2}-z_{1}=-A_{1}\left(z_{3}-z_{1}\right)$, отсюда следует, что $z_{1}=z_{2}$. Следовательно, при любом $\alpha>0$ мы имеем $z_{1}=z_{2}$, откуда $z_{1}=z_{2}=z_{3}=z_{4}$ на $I_{2}$.

2. В случае $|A|=k$ из (6) получим

$$
\begin{aligned}
& h_{2}=(k-1) f\left(h_{3}, \theta\right)+f\left(k f\left(h_{3}, \theta\right), \theta\right), \\
& h_{3}=(k-1) f\left(h_{2}, \theta\right)+f\left(k f\left(h_{2}, \theta\right), \theta\right) .
\end{aligned}
$$

Теперь докажем, что система (9) имеет только решения вида $h_{2}=h_{3}$. Рассмотрим случай $h_{2}>h_{3}$, тогда из (9) имеем

$$
h_{2}-h_{3}=(k-1)\left(f\left(h_{3}, \theta\right)-f\left(h_{2}, \theta\right)\right)+f\left(k f\left(h_{3}, \theta\right), \theta\right)-f\left(k f\left(h_{2}, \theta\right), \theta\right) .
$$

Легко проверить, что функция $f$ строго возрастает. Следовательно, равенство (10) не может иметь места, так как в левой части этого равенства стоит положительная величина, а в правой части - отрицательная. В случае $h_{2}<h_{3}(10)$ также не выполняется, следовательно, $h_{2}=h_{3}$, что дает трансляционно-инвариантные решения системы (6).

3. Доказательство третьего утверждения теоремы следует из леммы 3 , приведенной в п. 3.2. В данном случае необходимо проанализировать решения уравнения

$$
h=3 f(h, \theta)-f(4 f(h, \theta), \theta),
$$

которое получается при сужении оператора $W$ на $I_{3}$.

4. При $|A|=1, k>5$ из (6) получим

$$
h_{1}=g\left(h_{1}, \theta, k\right) \text {, }
$$

где $g(x)=g(x, \theta, k)=-f(k f(x, \theta), \theta)+(k-1) f(x, \theta), x \in \mathbb{R}$. Заметим, что $g(0)=0$ и $g$ - нечетная ограниченная функция. Из этих свойств следует, что если $g^{\prime}(0)>1$, 
то уравнение (11) имеет не менее трех решений. Легко проверить, что неравенство $g^{\prime}(0)>1$ эквивалентно $\theta_{-}<\theta<\theta_{+}$, при этом уравнение (6) имеет три решения

$$
\left( \pm h_{1}^{*}, \pm k f\left(h_{1}^{*}, \theta\right), \mp k f\left(h_{1}^{*}, \theta\right), \mp h_{1}^{*}\right), \quad(0,0,0,0) .
$$

ЗАмечАниЕ 1 . Меры $\mu^{ \pm}$и $\mu_{i}^{ \pm}, i=1,2$, являются $H_{A}$-слабо периодическими, и это дает новые меры Гиббса для модели Изинга. Все остальные меры, построенные в теореме, являются трансляционно-инвариантными.

ЗАмечание 2. Если $A \subset N_{k}$ таково, что $|A| \neq 1$ или $|A| \neq k$, то решение системы уравнений (6) вне инвариантов $I_{1}, I_{2}$ затруднительно. Даже на инварианте $I_{3}$ система (6) будет системой с двумя неизвестными, и ее решить нелегко.

3.2. Случай индекса 4. Пусть $H_{\left\{a_{1}\right\}}=\left\{x \in G_{k}: w_{x}\left(a_{1}\right)\right.$ - четное число $\}$, $G_{k}^{(2)}=\left\{x \in G_{k}:|x|-\right.$ четное число $\}$ и $G_{k}^{(4)}=H_{\left\{a_{1}\right\}} \cap G_{k}^{(2)}-$ соответствующий нормальный делитель индекса 4.

ЗАмЕчаниЕ 3. Среди всех нормальных делителей индекса 4 выбранный нами нормальный делитель $G_{k}^{(4)}$ удобен тем, что в этом случае из системы (4) мы получаем систему уравнений с 8 неизвестными, при этом для произвольного нормального делителя индекса 4 число неизвестных может достигать 16.

Рассмотрим фактор-группу $G_{k} / G_{k}^{(4)}=\left\{H_{0}, H_{1}, H_{2}, H_{3}\right\}$, где

$$
\begin{aligned}
& H_{0}=\left\{x \in G_{k}: w_{x}\left(a_{1}\right) \text { - четное число, }|x| \text { - четное число }\right\}, \\
& H_{1}=\left\{x \in G_{k}: w_{x}\left(a_{1}\right) \text { - нечетное число, }|x| \text { - четное число }\right\}, \\
& H_{2}=\left\{x \in G_{k}: w_{x}\left(a_{1}\right) \text { - четное число, }|x|-\text { нечетное число }\right\}, \\
& H_{3}=\left\{x \in G_{k}: w_{x}\left(a_{1}\right) \text { - нечетное число, }|x|-\text { нечетное число }\right\} .
\end{aligned}
$$

Тогда в силу (4) $G_{k}^{(4)}$-слабо периодическая совокупность $h$ имеет вид

$$
h_{x}=\left\{\begin{array}{lll}
h_{1}, & x \in H_{3}, & x_{\downarrow} \in H_{1}, \\
h_{2}, & x \in H_{1}, & x_{\downarrow} \in H_{3}, \\
h_{3}, & x \in H_{3}, & x_{\downarrow} \in H_{0}, \\
h_{4}, & x \in H_{0}, & x_{\downarrow} \in H_{3}, \\
h_{5}, & x \in H_{1}, & x_{\downarrow} \in H_{2}, \\
h_{6}, & x \in H_{2}, & x_{\downarrow} \in H_{1}, \\
h_{7}, & x \in H_{2}, & x_{\downarrow} \in H_{0}, \\
h_{8}, & x \in H_{0}, & x_{\downarrow} \in H_{2},
\end{array}\right.
$$


где $h_{i}, i=\overline{1,8}$, удовлетворяют системе уравнений

$$
\begin{aligned}
& h_{1}=(k-1) f\left(h_{2}, \theta\right)+f\left(h_{4}, \theta\right), \\
& h_{2}=(k-1) f\left(h_{1}, \theta\right)+f\left(h_{6}, \theta\right), \\
& h_{3}=k f\left(h_{2}, \theta\right), \\
& h_{4}=k f\left(h_{7}, \theta\right), \\
& h_{5}=k f\left(h_{1}, \theta\right), \\
& h_{6}=k f\left(h_{8}, \theta\right), \\
& h_{7}=(k-1) f\left(h_{8}, \theta\right)+f\left(h_{5}, \theta\right), \\
& h_{8}=(k-1) f\left(h_{3}, \theta\right)+f\left(h_{7}, \theta\right) .
\end{aligned}
$$

Эту систему можно переписать как $h=W(h)$, где отображение $W: \mathbb{R}^{4} \rightarrow \mathbb{R}^{4}$ задается следующими равенствами: $W(h)=h^{\prime}$, если

$$
\begin{aligned}
h_{1}^{\prime} & =(k-1) f\left(h_{2}, \theta\right)+f\left(k f\left(h_{7}, \theta\right), \theta\right), \\
h_{2}^{\prime} & =(k-1) f\left(h_{1}, \theta\right)+f\left(k f\left(h_{8}, \theta\right), \theta\right), \\
h_{7}^{\prime} & =(k-1) f\left(h_{8}, \theta\right)+f\left(k f\left(h_{1}, \theta\right), \theta\right), \\
h_{8}^{\prime} & =(k-1) f\left(h_{7}, \theta\right)+f\left(k f\left(h_{2}, \theta\right), \theta\right) .
\end{aligned}
$$

Нетрудно доказать следующую лемму.

ЛЕмма 1. Отображение $W$ имеет инвариантные множества

$$
\begin{array}{lll}
I_{1}=\left\{h \in \mathbb{R}^{4}: h_{1}=h_{2}=h_{7}=h_{8}\right\}, & I_{2}=\left\{h \in \mathbb{R}^{4}: h_{1}=h_{2}, h_{7}=h_{8}\right\}, \\
I_{3}=\left\{h \in \mathbb{R}^{4}: h_{1}=-h_{2}, h_{7}=-h_{8}\right\}, & I_{4}=\left\{h \in \mathbb{R}^{4}: h_{1}=h_{2}=-h_{7}=-h_{8}\right\}, \\
I_{5}=\left\{h \in \mathbb{R}^{4}: h_{1}=h_{7}, h_{2}=h_{8}\right\}, & I_{6}=\left\{h \in \mathbb{R}^{4}: h_{1}=-h_{7}, h_{2}=-h_{8}\right\}, \\
I_{7}=\left\{h \in \mathbb{R}^{4}: h_{1}=h_{7}=-h_{2}=-h_{8}\right\}, & I_{8}=\left\{h \in \mathbb{R}^{4}: h_{1}=h_{8}, h_{2}=h_{7}\right\}, \\
I_{9}=\left\{h \in \mathbb{R}^{4}: h_{1}=-h_{8}, h_{2}=-h_{7}\right\}, & I_{10}=\left\{h \in \mathbb{R}^{4}: h_{1}=h_{8}=-h_{2}=-h_{7}\right\} .
\end{array}
$$

Заметим, что сужение оператора $W$ на $I_{1}$ дает трансляционно-инвариантные меры, которые были изучены ранее.

Легко проверить, что, сужая оператор $W$ на остальные множества $I_{i}, i=2, \ldots, 10$, при некоторых дополнительных условиях на переменные, систему уравнений $W(h)=h$ можно привести к уравнениям с одной неизвестной, имеющим один из следующих видов:

$$
\begin{aligned}
& x=-(k-1) f(x, \theta)+f(k f(x, \theta), \theta), \\
& x=(k-1) f(x, \theta)-f(k f(x, \theta), \theta) .
\end{aligned}
$$

Уравнение (15) сводится к уравнению

$$
\left(u^{2}-1\right) P_{2 k-2}(u)=0,
$$

где $u=(z+\alpha) /(\alpha z+1)$ и $P_{2 k-2}(u)$ - симметрический многочлен степени $2 k-2$. Известно, что, положив $u+1 / u=\xi$, степень уравнения $P_{2 k-2}(u)=0$ можно уменьшить 
в два раза, т.е. привести это уравнению к $P_{k-1}(\xi)=0$, где $P_{k-1}(\xi)$ - вообще говоря, несимметрический многочлен степени $k-1$. Но при $k \geqslant 6$ уравнение $P_{k-1}(\xi)=0$ не решается в радикалах.

Рассмотрим случай $k=4$. В этом случае уравнение (17) имеет следующий вид:

$$
\left(u^{2}-1\right)\left(u^{6}-\alpha u^{5}+u^{4}+(1-\alpha) u^{3}+u^{2}-\alpha u+1\right)=0 .
$$

Лемма 2. При $\alpha>\alpha_{\text {сr }}^{\prime}=5 / 3$ уравнение (18) имеет три решения $u_{0}=1, u_{1}=$ $u_{*}, u_{2}=1 / u_{*} ;$ при $0<\alpha \leqslant 5 / 3$ уравнение (18) имеет единственное решение $u_{0}=1$.

ДокАзАТЕЛЬство. Для уравнения (18) $u=1$ является решением. Предположим, что $u \neq 1$. Положив $\xi=u+1 / u>2$, получим следующее уравнение:

$$
\xi^{3}-\alpha \xi^{2}-2 \xi+\alpha+1=0
$$

Детальный анализ этого уравнения показывает справедливость леммы.

Аналогично при $k=4$ из уравнения (16) имеем

$$
\alpha^{2}\left(u^{8}-1\right)-\alpha u\left(u^{6}-1\right)+u^{3}\left(u^{2}-1\right)=0 .
$$

Лемма 3. Существует критическое значение $\alpha_{\mathrm{cr}}(\approx 0.152)$ такое, что

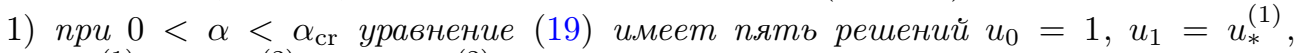
$u_{2}=1 / u_{*}^{(1)}, u_{3}=u_{*}^{(2)}, u_{4}=1 / u_{*}^{(2)}$;

2) при $\alpha=\alpha_{\text {сr }}$ уравнение (19) имеет три решения $u_{0}=1, u_{1}=u_{*}^{(1)}, u_{2}=1 / u_{*}^{(1)}$;

3) при $\alpha>\alpha_{\mathrm{cr}}$ уравнение (19) имеет единственное решение $u_{0}=1$.

ДокАЗАТЕльство. Для любого $\alpha>0$ значение $u=1$ является решением уравнения (19). Разделив уравнение (19) на $u^{2}-1$ и вводя обозначение $\xi=u+1 / u$, сводим (19) к уравнению

$$
\varphi(\xi)=\alpha^{2} \xi^{3}-\alpha \xi^{2}-2 \alpha^{2} \xi+\alpha+1=0 .
$$

Утверждения леммы следуют из легко проверяемых свойств функции $\varphi(\xi)$.

Решая уравнение $\varphi^{\prime}(\xi)=0$, получим $\xi=\xi_{*}=\left(1+\sqrt{1+6 \alpha^{2}}\right) / 3 \alpha$. Значения $\alpha_{\mathrm{cr}}$ находятся из уравнения $\varphi\left(\xi_{*}\right)=0$, т.е. из уравнения

$$
f(\alpha)=9 \alpha^{2}+27 \alpha-2-2\left(\sqrt{6 \alpha^{2}+1}\right)^{3}=0, \quad 0<\alpha<\frac{2}{5} .
$$

Заметим, что $f$ возрастает в интервале $(0,2 / 5)$. Так как $f(0)=-4, f(2 / 5)>0$, из монотонности $f$ следуют существование и единственность значения $\alpha_{\text {сr }}$ такого, что $f\left(\alpha_{\mathrm{cr}}\right)=0$. Расчеты на компьютере показывают, что $\alpha_{\mathrm{cr}} \approx 0.152$.

Объединяя леммы 2 и 3, получаем следующую теорему.

ТЕОРема 2. При $k=4$ существуют критические значения $\alpha_{\mathrm{cr}} \approx 0.152$ и $\alpha_{\mathrm{cr}}^{\prime}=$ $5 / 3$ maкuе, чmo 
1) при $0<\alpha<\alpha_{\text {cr }}$ существуют пять слабо периодических мер Гиббса $\mu_{0}, \tilde{\mu}_{1}^{ \pm}$, $\tilde{\mu}_{2}^{ \pm}$, которые соответствуют таким решениям системы уравнений (13):

$$
h_{x}=0, \quad \pm h_{x}^{(i)}=\left\{\begin{array}{lll} 
\pm h_{*}^{(i)}, & x \in H_{3}, & x_{\downarrow} \in H_{1}, \\
\pm h_{*}^{(i)}, & x \in H_{1}, & x_{\downarrow} \in H_{3}, \\
\pm 4 f\left(h_{*}^{(i)}, \theta\right), & x \in H_{3}, & x_{\downarrow} \in H_{0}, \\
\mp 4 f\left(h_{*}^{(i)}, \theta\right), & x \in H_{0}, & x_{\downarrow} \in H_{3}, \\
\pm 4 f\left(h_{*}^{(i)}, \theta\right), & x \in H_{1}, & x_{\downarrow} \in H_{2}, \\
\mp 4 f\left(h_{*}^{(i)}, \theta\right), & x \in H_{2}, & x_{\downarrow} \in H_{1}, \\
\mp h_{*}^{(i)}, & x \in H_{2}, & x_{\downarrow} \in H_{0}, \\
\mp h_{*}^{(i)}, & x \in H_{0}, & x_{\downarrow} \in H_{2}, \\
h_{*}^{(i)}=\frac{1}{2} \ln \frac{\alpha-u_{*}^{(i)}}{1-\alpha u_{*}^{(i)},} & i=1,2 ; &
\end{array}\right.
$$

2) при $\alpha=\alpha_{\text {сr }}$ существуют три слабо периодических меры Гиббса $\mu_{0}, \tilde{\mu}_{1}^{ \pm}$;

3) при $\alpha_{\mathrm{cr}}<\alpha \leqslant \alpha_{\text {cr }}^{\prime}$ существует одна мера Гиббса $\mu_{0}$, которая соответствует $h_{x}=0$;

4) при $\alpha>\alpha_{\text {сr }}^{\prime}$ существуют пять слабо периодических мер Гиббса $\mu_{0}, \mu_{*, 1}^{ \pm}, \mu_{*, 2}^{ \pm}$, которые соответствуют решениям вида

$$
h_{x}=0, \quad \pm \bar{h}_{x}^{(1)}=\left\{\begin{array}{lll} 
\pm \bar{h}_{*}^{(1)}, & x \in H_{3}, & x_{\downarrow} \in H_{1}, \\
\mp \bar{h}_{*}^{(1)}, & x \in H_{1}, & x_{\downarrow} \in H_{3}, \\
\mp 4 f\left(\bar{h}_{*}^{(1)}, \theta\right), & x \in H_{3}, & x_{\downarrow} \in H_{0}, \\
\pm 4 f\left(\bar{h}_{*}^{(1)}, \theta\right), & x \in H_{0}, & x_{\downarrow} \in H_{3}, \\
\pm 4 f\left(\bar{h}_{*}^{(1)}, \theta\right), & x \in H_{1}, & x_{\downarrow} \in H_{2}, \\
\mp 4 f\left(\bar{h}_{*}^{(1)}, \theta\right), & x \in H_{2}, & x_{\downarrow} \in H_{1}, \\
\pm \bar{h}_{*}^{(1)}, & x \in H_{2}, & x_{\downarrow} \in H_{0}, \\
\mp \bar{h}_{*}^{(1)}, & x \in H_{0}, & x_{\downarrow} \in H_{2},
\end{array}\right.
$$

на инварианте $I_{7}$ и решениям вида

$$
\pm \bar{h}_{x}^{(1)}=\left\{\begin{array}{lll} 
\pm \bar{h}_{*}^{(1)}, & x \in H_{3}, & x_{\downarrow} \in H_{1}, \\
\mp \bar{h}_{*}^{(1)}, & x \in H_{1}, & x_{\downarrow} \in H_{3}, \\
\mp 4 f\left(\bar{h}_{*}^{(1)}, \theta\right), & x \in H_{3}, & x_{\downarrow} \in H_{0}, \\
\mp 4 f\left(\bar{h}_{*}^{(1)}, \theta\right), & x \in H_{0}, & x_{\downarrow} \in H_{3}, \\
\pm 4 f\left(\bar{h}_{*}^{(1)}, \theta\right), & x \in H_{1}, & x_{\downarrow} \in H_{2}, \\
\pm 4 f\left(\bar{h}_{*}^{(1)}, \theta\right), & x \in H_{2}, & x_{\downarrow} \in H_{1}, \\
\mp \bar{h}_{*}^{(1)}, & x \in H_{2}, & x_{\downarrow} \in H_{0}, \\
\pm \bar{h}_{*}^{(1)}, & x \in H_{0}, & x_{\downarrow} \in H_{2}
\end{array}\right.
$$

на инварианте $I_{10}$. 
ЗАмЕчАниЕ 4. Компьютерный анализ показывает, что уравнение $g(x, \theta, k)=$ $-f(k f(x, \theta) ; \theta)+(k-1) f(x, \theta)=x$ имеет единственное решение $x=0$ при $k=1,2,3$; если $k \geqslant 4$, то существуют значения параметра $\alpha$, при которых это уравнение имеет пять решений. Точнее, компьютерный анализ показывает, что пункт 2 теоремы 1 и результаты теоремы 2 верны для всех $k \geqslant 4$, т.е. независимо от значения $k \geqslant 4$ существуют не более пяти $H_{A}$-слабо периодических мер Гиббса.

ЗАмечАние 5 . Меры $\tilde{\mu}_{i}^{\mp}, i=1,2$, совпадают с $H_{\left\{a_{1}\right\}}$-слабо периодическими мерами теоремы 1 в случае $A=\left\{a_{1}\right\}$. Меры $\mu_{*, i}^{\mp}, i=1,2$, являются $G_{k}^{(4)}$-слабо периодическими, но не совпадают с $H_{A}$-слабо периодическими мерами, построенными в теореме 1 .

ЗАмечание 6. Новые меры Гиббса, описанные в теоремах 1 и 2, дают возможность описать континуальное множество непериодических мер Гиббса, отличных от ранее известных.

\section{4. ОБСУЖДЕНИЕ. ОТКРЫТЫЕ ПРОБЛЕМЫ}

Результаты настоящей работы открывают новый факт для модели Изинга: известно, что для моделей с конечным радиусом взаимодействия существуют не более трех периодических (нетрансляционно-инвариантных) мер Гиббса [4], [5], [7], [10]; в нашем случае число слабо периодических мер оказалось равным пяти.

Функции $h_{x}^{(i)}$, определенные в п. 1 теоремы 2 , существенно отличаются от функций $\bar{h}_{x}^{(1)}$, определенных в п. 4 этой теоремы. Слабая (H-слабая) периодичность функции $h_{x}$ означает, что значение функции $h_{x}$ зависит только от класса принадлежности $x$ и от класса принадлежности $x_{\downarrow}$. Такая зависимость впервые была показана в работе [3], но в этой работе не описаны слабо периодические решения.

Сформулируем вопросы, решение которых оказалось достаточно сложным, и требуется их рассмотрение в дальнейшем.

1. Существуют ли другие инвариантные множества операторов, заданных равенствами (7) и (14)?

2. Существуют ли неподвижные точки вне инвариантных множеств?

3. Как доказать замечание 4 после теоремы 2 ?

4. Как описать слабо периодические меры Гиббса для нормальных делителей индекса больше 4 ?

5. Как описать такие меры для других моделей, например для модели Поттса, SOS-модели и т.п.?

Благодарности. У.А. Розиков благодарит Institut des Hautes Études Scientifiques (Bures-sur-Yvette, France) за финансовую поддержку пребывания в октябредекабре 2006 г., а также School of Mathematical Sciences (Lahore, Pakistan) за финансовую поддержку пребывания в феврале-мае 2007 г. Авторы благодарят рецензента, замечания которого улучшили стиль работы. 


\section{Список литературы}

[1] П. М. Блехер, Н. Н. Ганиходжаев, ТВП, 35:2 (1990), 220-230.

[2] F. Spitzer, Ann. Probab., 3:3 (1975), 387-398.

[3] S. Zachary, Ann. Probab., 11:4 (1983), 894-903.

[4] Н. Н. Ганиходжаев, У. А. Розиков, ТМФ, 111:1 (1997), 109-117.

[5] У. А. Розиков, ТМФ, 112:1 (1997), 170-175.

[6] У. А. Розиков, ТMФ, 118:1 (1999), 95-104.

[7] U. A. Rozikov, Yu. M. Suhov, Inf. Dimens. Anal. Quantum Probab. Relat. Top., 9:3 (2006), 471-488.

[8] J. Martin, U. A. Rozikov, Yu. M. Suhov, J. Nonlinear Math. Phys., 12:3 (2005), 432-448.

[9] У. А. Розиков, Сиб. матем. журн., 39:2 (1998), 427-435.

[10] F. M. Mukhamedov, U. A. Rozikov, J. Statist. Phys., 114:3-4 (2004), 825-848.

[11] У. А. Розиков, Ш. А. Шоюсупов, ТМФ, 149:1 (2006), 18-31.

[12] Э. П. Норматов, У. А. Розиков, Матем. заметки, 79:3 (2006), 434-444.

Поступила в редакцию 26.07.2007, после доработки 23.10.2007 